\title{
Delirium Risk Factors in Hospitalized Patient: a Comprehensive Evaluation of Underlying Diseases and Medications in Different Wards of a Large Urban Hospital Center in Iran
}

\author{
Mohammad Arbabi \\ Tehran University of Medical Sciences \\ Elham Ziaei \\ Tehran University of Medical Science \\ Behnam Amini \\ Tehran University of Medical Science \\ Hamidreza Ghadimi \\ Tehran University of Medical Science
}

Fatemeh Radishi

Tehran University of Medical Science

Narges Shohanizad

Azad University of Medical Science

Soroush Moradi

Tehran University of Medical Sciences

Alireza Beikmarzehei

Tehran University of Medical Sciences

Alireza Hasanzadeh

Tehran University of Medical Sciences

Amirhossein Parsaei ( $\nabla$ ah-parsaei@alumnus.tums.ac.ir)

Tehran University of Medical Sciences

\section{Research Article}

Keywords: Delirium, confusion, hospitalization, dementia, neoplasms, neurological diseases

Posted Date: February 7th, 2022

DOI: https://doi.org/10.21203/rs.3.rs-1167386/v1

License: (1) This work is licensed under a Creative Commons Attribution 4.0 International License.

Read Full License 
Page 2/15 


\section{Abstract}

Background: Delirium is a neurobehavioral syndrome, which is characterized by fluctuation of mental status, disorientation, confusion and inappropriate behavior and it is prevalent among hospitalized patients. Recognizing modifiable risk factors of delirium is the key point for improving our preventive strategies and restraining its devastating consequences. The present study investigated a wide range of possible predisposing factors of delirium, mainly focused on underlying diseases and mediations, of hospitalized patients in the different wards of a general hospital.

Method: In a prospective, observational trial, we investigated 220 patients who had been admitted to the internal, emergency, surgery and hematology-oncology departments. We employed the Confusion Assessment Method (CAM) questionnaire, The Richmond Agitation Sedation Scale (RASS), the General Practitioner Assessment of Cognition (GPCOG), demographic questionnaire, patient interviews and medical records. Multivariate logistic regression models were used to analyze predictive value of medications and underlying diseases for daily transition to delirium.; demographics were analyzed using univariate analysis to identify those independently associated with delirium.

Results: 220 patients were enrolled; the emergency department had the most incident delirium (\%31.3) and the surgery section had the least (\%2.4); delirium was significantly correlated with older ages and sleep disturbance. Among multiple underlying diseases and the medications evaluated in this study, we found that history of dementia, neurological diseases and malignancies increase the odds of transition to delirium and the use of anticoagulants decreases the incident delirium.

Conclusion: Approximately, 1 out of 10 overall patients developed delirium; Considering underlying diseases and the medications as the predisposing factors of delirium would help to better predict those at risk.

\section{Introduction}

Delirium is a neurobehavioral syndrome, which is characterized by fluctuation of mental status, disorientation, confusion and inappropriate behavior[3]. Disturbance of serum metabolites , neuroendocrine systems and neurotransmitters - specially cholinergic and dopaminergic systems- are assumed to play roles in delirium's pathophysiology by disrupting the neuronal activity[4-6]. The point prevalence of the delirium in patients over the age of 65 is more than $7.8 \%[7]$ and its incidence rate in hospitalized patients differs based on the underlying condition: it ranges from $5 \%$ to $87 \%[8,9]$-with overall higher incidences in intensive care unit (ICU) patients.

Well known risk factors of delirium consist of: cognitive impairment, dementia, hypertension, depression, history of stroke and older ages[10,11]. Metabolic diseases such as hepatic encephalopathy, neuroendocrine disorders such as diabetes and certain classes of drugs like benzodiazepines, high dose narcotics, and anticholinergic medications have been linked to increased risk of delirium $[12,13]$.Other known risk factors of delirium that by decreasing the perception of environment, increase the odds of 
developing delirium consisted of: Inappropriate ambient light, sleep disorders and visual and hearing impairments.

Delirium increases the mortality rate, lengthen the hospitalization and places a heavy burden on hospitals and long-term care facilities[14]; delirium is also hard to diagnose and treat. So, to prevent delirium, its prevalence in each medical section and its associated risk factors should be characterized properly. The purpose of our study is to provide means for the early diagnosis and prevention of this syndrome among hospitalized patients by identifying the possible risk factors; so in a prospective, observational cohort study of 220 patients of several medical sections, we determined the incidence of delirium and evaluated its associated risk factors.

\section{Methods}

We enrolled patients from June 17, 2019 through September 2,2019. All patients admitted to internal, surgical, emergency and hematology-oncology wards of Imam Khomeini hospital, Tehran, Iran were evaluated and 220 patients were included to the study. Enrollment criteria included: 1- all patients 18 years or older, 2- not requiring mechanical ventilation. Exclusion criteria were as follows: 1- symptoms of withdrawal or intoxication (Based on clinical evaluations and Paraclinical tests and interviews with patient companions) 2- moribund patients not expected to survive $>24$ hrs Decreased level of consciousness (RASS score -4 or -5 , which considered as coma) $[15,16] 3$ - Inability to understand or speak Persian. Patients were followed up until they were discharged from the hospital or developed delirium.

As shown in figure 1, at first, baseline demographics as well as information pertaining to known risk factors for delirium were obtained through patient interviews and medical records. These included age, sex, city of residency, level of education, marital status, employment status, number of family members and the patient's general status information such as vision and hearing impairment and also access to visual and auditive aids if needed, quality and quantity of sleep, underlying diseases and medications.

Patients were evaluated once daily for delirium: at first, the level of consciousness was measured by The Richmond Agitation Sedation Scale (RASS) and if the RASS score was above $-4(-3$ to +4$)$ - the RASS score of -5 or -4 is considered as coma- then the confusion assessment method (CAM) questionnaire[1, 2] and the General Practitioner Assessment of Cognition (GPCOG) test was employed.

The Richmond Agitation Sedation Scale (RASS) is a valid instrument to measure the level of arousal; it is a 10-point scale ranging from -5 to +4 : score of 0 represents a calm and alert state. Positive RASS scores indicate aggressive status and the negative RASS scores imply decreased levels of consciousness. The CAM questionnaire assesses the presence, severity, and fluctuation of 9 delirium features: acute onset, inattention, disorganized thinking, altered level of consciousness, disorientation, memory impairment, perceptual disturbances, psychomotor agitation or retardation, and altered sleep-wake cycle, and GPCOG test is used to evaluate patients for cognitive impairments and it is consisted of two components: a cognitive assessment and an informant questionnaire. The cognitive assessment includes nine items, 
each correct answer is valid one point leading to a maximum score of 9; results $=9$ or $<5$ considered to be cognitively intact or impaired, respectively. Informant questionnaire only considered necessary if the cognitive test is equivocal (5-8 scores), the informant questionnaire query an informant (person who knows the patient well) six historical questions, a score of 0-3 indicates cognitive impairment and requires further investigations.

\section{Statistical analysis}

Chi square test was used to investigate the correlation between nominal and ordinal variables, after that the normality of variables was examined by Kolmogorov-Smirnov test; independent t-test was performed to investigate the relationship between numeric variables with normal distribution and delirium. Mann Whitney $U$ statistical test was used to evaluate the difference between numeric variables with abnormal distribution. Adjusted odds ratios $(\mathrm{OR})$ and confidence intervals $(\mathrm{Cl})$ were calculated for factors with significant univariate correlations.

Logistic regression was used to evaluate the predictive value of the significant risk factors, and the Hosmer and Lemeshow test was used to assess the fit of the risk prediction models; delirium was considered as dependent variable and the significant risk factors as independent variables $(\mathrm{x})$ and the risk factors were compared one by one with the dependent variable (delirium). Regression coefficients with 95\%-confidence intervals $(\mathrm{Cl})$ and the corresponding $\mathrm{p}$-values were calculated for each risk factor. Moreover, odds ratios (OR) with 95\%-Cl were determined in the logistic regression.

Since, some patients had been concurrently afflicted by multiple diseases or been used multiple drugs, with regards to confounding effects of underlying diseases or drugs on each other, justified odds ratios were calculated. However, due to the low number of patients with hyperlipidemia and liver disorders, the adjusted odds ratios were not calculated in these two subgroups.

All data were analyzed using the 11th version of Stata and the first-order error(a) equal to 0.05 was considered to be statistically significant.

\section{Results}

Of the 220 patients studied, 114 were male $(51.9 \%)$ and the average age was 59.3 years $(S D=13)$. Of these patients, 112 patients (50.9\%) hospitalized in the internal ward, $83(37.7 \%)$ in the surgery section, $16(7.2 \%)$ in the emergency department and $9(4.09 \%)$ in hematology-oncology ward. The overall prevalence of delirium was $10 \%$, and we obtained the highest prevalence in the emergency department $(31.3 \%)$ and the least in the surgical section (2.4\%). Patients developed delirium on average 4 days after hospitalization, that it was ranged from 1 to 14 days (median= $2, \mathrm{IQR}=3$ ).

The overall prevalence of delirium in the study was $10 \%$, which it differed in various sections of the hospital; we obtained the incidence of delirium as follows: emergency department $31.3 \%$, hematologyoncology ward $22.2 \%$, internal ward $11.6 \%$, and surgery section $2.4 \%$ (Table 1 ). 
Table 1. Prevalence of delirium by ward

\begin{tabular}{|llll|}
\hline Ward & affected & Not affected & Prevalence \\
\hline Internal & 13 & 99 & $11.6 \%$ \\
\hline Surgery & 2 & 81 & $2.4 \%$ \\
\hline Emergency & 5 & 11 & $31.3 \%$ \\
\hline Hematology-Oncology & 2 & 7 & $22.2 \%$ \\
\hline Total & $\mathbf{2 2}$ & $\mathbf{2 2 0}$ & $\mathbf{1 0 \%}$ \\
\hline
\end{tabular}

By analyzing demographic indicators, we found significant association between age ( $p$-value $=0.014)$ and the incidence of delirium, but there was no significant correlation between incidence of delirium and gender ( $p$-value= 0.857), education ( $p$-value= 0.414$)$, city of residency ( $p$-value= 0.386$)$, employment status ( $p$-value $=0.395)$, living with family members $(p$-value $=0.178)$ and marital status $(p$-value $=0.093)$.

By examining the clinical signs and using the information obtained from the interview, we found that the incidence of delirium was significantly associated with dementia ( $p$-value $=0.008)$ and had a negative correlation with the quantity of sleep hours ( $p$-value $<0.0001)$ and its quality ( $p$-value< 0.0001$)$. However, there was no significant correlation between type of vision and hearing impairment ( $p$-value $=0.336,1.000$ respectively), use and access to hearing aids ( $p$-value $=1.000,1.000$ respectively) and visual aids ( $p$-value= $0.052,0.274$ respectively), sleep habits $(p$-value $=1.000)$, and visual and hearing health status ( $p$-value $=$ $0.056,0.795$ respectively)(Table 2 ).

Table 2. Demographics and delirium interview information of the patients 


\begin{tabular}{|c|c|c|c|c|c|c|c|}
\hline \multirow[t]{3}{*}{ Factors } & \multicolumn{2}{|l|}{ Delirium } & \multirow{3}{*}{$\begin{array}{l}\mathrm{P}- \\
\text { value }\end{array}$} & \multirow[t]{3}{*}{ Factors } & \multicolumn{2}{|l|}{ Delirium } & \multirow{3}{*}{$\begin{array}{l}\text { P- } \\
\text { value }\end{array}$} \\
\hline & & $\begin{array}{l}\text { Not- } \\
\text { affected }\end{array}$ & & & & $\begin{array}{l}\text { Not- } \\
\text { affected }\end{array}$ & \\
\hline & No. (\%) & No. (\%) & & & No. (\%) & No. (\%) & \\
\hline Vision & & & & Sex & & & \\
\hline Intact & $3(13.64)$ & $67(34)$ & 0.056 & Male & $11(50)$ & $103(52)$ & 0.857 \\
\hline $\begin{array}{l}\text { Impaired or } \\
\text { blind }\end{array}$ & 19(86.36) & $130(66)$ & & Female & $11(50)$ & $95(48)$ & \\
\hline $\begin{array}{l}\text { Type of } \\
\text { visual loss }\end{array}$ & & & & $\begin{array}{l}\text { City of } \\
\text { residence }\end{array}$ & & & \\
\hline Partial & 18(94.7) & $128(98.5)$ & 0.336 & Capital & $11(50)$ & $80(40.4)$ & 0.386 \\
\hline Total & $1(5.3)$ & $2(1.5)$ & & Others & $11(50)$ & $118(59.6)$ & \\
\hline $\begin{array}{l}\text { Use of } \\
\text { eyeglasses }\end{array}$ & & & & Education & & & \\
\hline Yes & 6(33.3) & 75(57.7) & 0.052 & Illiterate & $11(50)$ & 75 (37.9) & 0.414 \\
\hline No & 13(66.7) & $55(42.3)$ & & $\begin{array}{l}\text { Under } \\
\text { diploma }\end{array}$ & $9(40.9)$ & $81(40.9)$ & \\
\hline $\begin{array}{l}\text { Access to } \\
\text { eyeglasses }\end{array}$ & & & & Diploma & $0(0)$ & $21(10.6)$ & \\
\hline Yes & $3(16.7)$ & 41(31.5) & 0.274 & $\begin{array}{l}\text { University } \\
\text { degree }\end{array}$ & $2(9.1)$ & $21(10.6)$ & \\
\hline No & 15(83.3) & $89(68.5)$ & & $\begin{array}{l}\text { Marital } \\
\text { status }\end{array}$ & & & \\
\hline Hearing & & & & Married & $\begin{array}{l}13 \\
(59.1)\end{array}$ & $152(76.8)$ & 0.093 \\
\hline Intact & 17(77.3) & $148(74.7)$ & 0.795 & Single & $0(0)$ & $5(2.5)$ & \\
\hline $\begin{array}{l}\text { Impaired or } \\
\text { deaf }\end{array}$ & $5(22.7)$ & $50(25.3)$ & & Widow & $9(40.9)$ & 35 (17.7) & \\
\hline $\begin{array}{l}\text { Type of } \\
\text { hearing loss }\end{array}$ & & & & Divorced & $0(0)$ & $6(3)$ & \\
\hline Partial & $5(100)$ & $47(94.2)$ & 1.000 & $\begin{array}{l}\text { Employment } \\
\text { status }\end{array}$ & & & \\
\hline Total & $0(0)$ & $3(5.8)$ & & Employed & 3 (13.6) & $37(18.7)$ & 0.395 \\
\hline $\begin{array}{l}\text { Use of } \\
\text { hearing aid }\end{array}$ & & & & Retired & $2(9.1)$ & 38 (19.2) & \\
\hline
\end{tabular}




\begin{tabular}{|c|c|c|c|c|c|c|c|}
\hline Yes & $0(0)$ & $5(9.8)$ & 1.000 & Unemployed & $\begin{array}{l}17 \\
(77.3)\end{array}$ & $123(62.1)$ & \\
\hline No & $5(100)$ & $45(90.2)$ & & Insurance & & & \\
\hline $\begin{array}{l}\text { Access to } \\
\text { hearing aid }\end{array}$ & & & & Yes & $\begin{array}{l}12 \\
(54.6)\end{array}$ & $122(61.6)$ & 0.519 \\
\hline Yes & $0(0)$ & $3(5.9)$ & 1.000 & No & $\begin{array}{l}10 \\
(45.4)\end{array}$ & $76(38.4)$ & \\
\hline No & $5(100)$ & $47(94.1)$ & & $\begin{array}{l}\text { Living } \\
\text { with family } \\
\text { members }\end{array}$ & & & \\
\hline Dementia & & & & Yes & $\begin{array}{l}19 \\
(86.4)\end{array}$ & 186 (93.9) & 0.178 \\
\hline Yes & $3(13.6)$ & $2(1)$ & 0.008 & No & $3(13.6)$ & $12(6.1)$ & \\
\hline No & 19(86.4) & 196(99) & & Age & $\begin{array}{l}65.7^{\star} \\
\left(12.8^{\star \star}\right)\end{array}$ & $58.6\left(12.8^{\star \star}\right)$ & 0.014 \\
\hline \multicolumn{8}{|l|}{$\begin{array}{l}\text { Comfortable } \\
\text { sleep }\end{array}$} \\
\hline Yes & $2(9.1)$ & $122(61.6)$ & 0.0001 & & & & \\
\hline No & $20(90.9)$ & $76(38.4)$ & & & & & \\
\hline \multicolumn{8}{|l|}{$\begin{array}{l}\text { Adequate } \\
\text { sleep }\end{array}$} \\
\hline Yes & $3(13.6)$ & $171(86.3)$ & 0.0001 & & & & \\
\hline No & 19(86.4) & $27(13.6)$ & & & & & \\
\hline \multicolumn{8}{|l|}{ Sleep habits } \\
\hline Yes & $1(4.5)$ & $15(7.6)$ & 1.000 & & & & \\
\hline No & $21(95.5)$ & $183(92.4)$ & & & & & \\
\hline
\end{tabular}

*mean age of the group

**standard deviation

Since some patients had several diseases at the same time, adjusted statistical ratios were used to eliminate this confounding factor and based on that, we found significant association between delirium and underlying dementia, neurological diseases and malignancies. but, didn't find any significant correlation between delirium and diabetes, hypertension, hyperlipidemia, cardiovascular disease, renal, hepatic, Pulmonary and infectious diseases(Table 3).

Table 3. Analyzing the underlying diseases and medications of the patients for delirium 


\begin{tabular}{|c|c|c|c|c|c|}
\hline Underlying disease & adjusted & & Drug group & adjusted & \\
\hline & $\mathrm{OR}(\mathrm{Cl})$ & P-value & & $\mathrm{OR}(\mathrm{Cl})$ & $P$-value \\
\hline Dementia & $10.6(1.2-93.9)$ & 0.034 & Anti-diabetics & $0.5(0.1-2.3)$ & 0.152 \\
\hline Diabetes & $2.6(6.9-7.0)$ & 0.072 & Anti-hypertensives & $0.7(0.1-3.7)$ & 0.432 \\
\hline Hypertension & $1.1(3.0-5.3)$ & 0.843 & Diuretics & $0.4(0.1-1.3)$ & 0.124 \\
\hline Hyperlipidemia & - & - & Antibiotics & $2.3(0.5-9.8)$ & 0.086 \\
\hline $\begin{array}{l}\text { Cardiovascular } \\
\text { disease }\end{array}$ & $0.9(1.2-3.0)$ & 0.874 & Anti-hyperlipidemics & $0.6(0.1-2.8)$ & 0.361 \\
\hline Kidney disease & $1.1(2.2-6.0)$ & 0.951 & Analgesics & $1.4(0.3-5.6)$ & 0.463 \\
\hline Liver disease & - & - & Anti-coagulants & $0.3(0.0-1.9)$ & 0.029 \\
\hline Neurologic disease & $8.1(6.1-31.2)$ & 0.002 & Chemotherapy & $0.2(0.1-3.2)$ & 0.078 \\
\hline Malignancies & $3.3(8.0-10.1)$ & 0.048 & Anti-convulsant & $0.2(0.1-3.2)$ & 0.078 \\
\hline Lung disease & $1.4(7.2-12.0)$ & 0.737 & Sedatives & $2.1(0.16-3.5)$ & 0.481 \\
\hline Infectious disease & $1.2(5.9-15.0)$ & 0.899 & Opioids & 0.9 & 0.861 \\
\hline
\end{tabular}

we found a significant correlation between anticoagulant use and delirium. No significant association was found between delirium and use of anti-diabetic, anti-hypertensives, diuretics, antibiotics, antihyperlipidemics, analgesics, chemotherapy agents, sedatives, opioids and anti-convulsants; nevertheless, antibiotics $(p$-value $=0.086)$, chemotherapy agents $(p$-value $=0.078)$ and anticonvulsants $(p$-value $=0.078)$ had a near significant $p$-value and needed to be further investigated.

\section{Discussion}

Our study is a prospective, observational trial that evaluated the incidence rate of delirium and its contributing risk factors in 220 patients admitted to the internal, emergency, surgery and hematologyoncology departments.; first, we found that 1 out of 10 overall patients developed delirium; the emergency department had the most incident delirium (\%31.3) and the surgery section had the least (\%2.4). Second, by assessing the demographic indicators and general status information of included patients, we found a meaningful positive correlation between the incident delirium, and older ages and sleep disturbance; but we found no significant correlation between delirium and gender, employment status, sleep habits, living with family members, marital status, educational degree, visual or auditory impairment and access to visual and hearing aids. Third, by utilizing multivariate regression analysis for multiple underlying diseases and the medications, we found that history of dementia, neurological diseases and malignancies increase the odds of transition to delirium and the use of anticoagulants decrease the 
incident delirium, but the correlation of delirium and other underlying diseases like diabetes, hypertension, cardiovascular disease, renal, Pulmonary and infectious diseases and also other medications wasn't meaningful.

The $10 \%$ incidence of delirium in this study is concordant with previous reports[17] and is lower than of the higher incidences reported by most of the other studies[17, 18]. This salient discrepancy might be originated in differences in patient characteristics (e. g., the average age, the severity of underlying condition, type of the diseases), the screening instrument and its application and assuming the Druginduced sedation and medication-associated coma as delirium. And also the differences of delirium prevalence seen in our under study wards, could be due to differences in follow-up length, the patient characteristics and their specific medications [19]; the lower incidence of delirium in the surgical ward comparing to the internal ward, could be due to younger ages and also, most of them were at non-urgent surgery condition with a good health background.

The sleep hours and its quality, as reported by previous studies, have a strong correlation with delirium. The disturbed neurotransmission underlies this relationship: the REM cycles of the sleep adjust the acetyl choline and dopamine neurotransmission; and the both cholinergic and dopaminergic systems are reported to be dysregulated in the delirium state [20,21]. Sleep habits, like the sleep time or afternoon naps, have not been associated with incident delirium. Baseline cognitive deficits were associated with an increased risk of developing delirium[4]; The presence of dementia and neurological disorders, through decreasing cerebral oxidative metabolism, cholinergic deficiency and inflammation increase the odds of developing delirium[22].

Malignancies are known to increase the chances of developing delirium through their adverse effects on the immune system, the blood-brain barrier, and the nervous system[23]. The number of patients with malignancy involved in this study was not enough to evaluate the specific subtypes of malignancies for delirium, but according to previous studies, the patients with primary or secondary CNS tumors, cancers with paraneoplastic neurological features and terminal cancers are at higher risks to develop delirium[24, 25]; however, taking the chemotherapy medications that were reported as predisposing factor for delirium in previous studies, were not associated with higher risks of delirium in our study. Because of insufficient number of the patients with hyperlipidemia and liver disease, we weren't able to analyze these two variables; according to previous studies, hyperlipidemia plays a protective role by strengthening the bloodbrain barrier and liver diseases by disturbing plasma metabolites and electrolytes are risk factors for delirium[26-28].

In spite of other studies reported a relationship between delirium and diabetes[29], we couldn't find an association between them; diabetes mellitus is a chronic disease that is often accompanied by other diseases and also, the diabetic patients receive numerous medications; hence, to find out whether diabetes increase the odds of developing delirium, these all covariates should be evaluated cautiously. Siew et al.[30] reported that acute kidney injury increases the odds of developing delirium; but we only evaluated the chronic kidney diseases in this study and didn't find a meaningful relationship. Infectious 
diseases like urinary tract infections were reported to increase the chance of delirium [31]; but infectious diseases weren't associated to delirium in our study that could be due to the lack categorization and also, younger ages of our participants. The lack of relationship between delirium and cardiac and pulmonary disorders, which we obtained, resembles previous studies[26].

Assessments of the role of medications in developing delirium by previous studies is limited and inconsistent[32, 33]. So, we investigated the role of a wide range of prescribed drugs for developing delirium. The use of anticoagulants (warfarin, heparin and enoxaparin) decreased the odds of developing delirium that resembles previous studies about warfarin[34]. However, we couldn't find any relationship between delirium and other drugs, but the level of significance of chemotherapy agents and antibiotics were marginal and both increased the odds of developing delirium.

Several limitations of this investigation warrant consideration: first, our sample size limited our ability to evaluate some underlying diseases like liver diseases and hyperlipidemia and also specific subtypes of diseases like malignancies or drugs like chemotherapy medications and antibiotics. Second, we did not consider any laboratory values in our study because the diagnostic laboratory values for delirium are still in advances[35-37]. Third, we evaluated delirium once daily; based on the fluctuating nature of delirium, it's possible that some cases have been missed; by assessing patient's cognitive status more frequently (every 4-8 hours), this bias would be removed, but this task is difficult to accomplish in a research setting due to resource and time constraints and also it is burdensome to patients. Fourth, we investigated numerous covariates that were deemed relevant a priori; so, other covariates that weren't measured might have affected our results. The strengths of this study lay in its diverse sample of medical patients with different types of conditions.

\section{Conclusion}

In summary, we investigated a wide range of medical and demographical factors to find the predisposing factors of delirium; this study documents the prevalence and risk factors of delirium in a prospective study of patients admitted in different sections of a referral hospital. The adverse outcomes of developing delirium are burdensome for healthcare system and is accompanied by decreased quality of life and increased mortality and morbidity of hospitalized patients. Therefore, recognizing the predisposing factors of delirium is the first step to prepare the healthcare systems to decrease the incidence and restrain the consequences. Future studies would need to explore that by which molecular and biological mechanisms, the known risk factors of delirium increase its occurrence and also, more interventional studies in this area are needed to strengthen our preventive and therapeutic strategies that at the moment, are not effective enough to prevent or cure delirium in most of the patients.

\section{Declarations}


The current study was authorized by the ethical committee at Tehran University of Medical Sciences and followed the requirements of the 2013 edition of the Helsinki Declaration. All participants completed and signed a written informed consent form, and all personal information was considered confidential.

\section{CONSENT FOR PUBLICATION}

Not applicable.

\section{AVAILABLITY OF DATA AND MATERIALS}

Researchers can obtain the datasets used in the current work from the Tehran University of Medical Sciences upon request. The data sharing policies in the Tehran University of Medical Sciences follow local regulations of our institution.

\section{COMPETING INTERESTS}

The authors of this article declare no competing interest regarding current research.

FUNDING

This research received no specific grant from any funding agency in the public, commercial, or not-forprofit sectors. The content is solely the responsibility of the authors.

\section{AUTHOR'S CONTRIBITUTIONS}

M.A., S.M., and E.Z. all contributed to the article's concept and early design. The measurements and data collection were done by A.B, B.A. and H.G. The technique, data interpretation, and preliminary analysis were all done by F.R. and M.A. E.Z. and A.P. assisted in the control and planning of the project. N.S. and S.M. examined the data, completed the final analysis, authored the text, and created the figures. A.H, B.A., H.G., F. R., and N. S. contributed to the interpretation of the findings and helped with the article. All authors discussed the findings and provided feedback on the work before approving the final version for publication.

\section{ACKNOWLEDGEMENTS}

We would like to thanks Dr Ghasem Rahmani for their motivation and clear guidance on the project.

\section{References}

1. Arbabi, M., et al., Validity and reliability of the Persian version of the confusion assessment method for intensive care units. Nursing Practice Today, 2019. 6(3): p. 123-132.

2. Ely, E.W., et al., Evaluation of delirium in critically ill patients: validation of the Confusion Assessment Method for the Intensive Care Unit (CAM-ICU). Critical care medicine, 2001. 29(7): p. 1370-1379. 
3. Morandi, A., et al., Understanding international differences in terminology for delirium and other types of acute brain dysfunction in critically ill patients. Intensive care medicine, 2008. 34(10): p. 1907.

4. Maldonado, J.R., Delirium pathophysiology: An updated hypothesis of the etiology of acute brain failure. International journal of geriatric psychiatry, 2018. 33(11): p. 1428-1457.

5. Nguyen, D.N., et al., High prolactin levels are associated with more delirium in septic patients. Journal of critical care, 2016. 33: p. 56-61.

6. Hshieh, T.T., et al., Cholinergic deficiency hypothesis in delirium: a synthesis of current evidence. The Journals of Gerontology Series A: Biological Sciences and Medical Sciences, 2008. 63(7): p. 764-772.

7. Scott, J., et al., A short report on point prevalence of delirium in hospitalised older adult patients in Auckland, New Zealand. Journal of Geriatric Care and Research, 2019. 6(1).

8. McNicoll, L., et al., Delirium in the intensive care unit: occurrence and clinical course in older patients. Journal of the American Geriatrics Society, 2003. 51(5): p. 591-598.

9. Abawi, M., et al., Postoperative delirium in individuals undergoing transcatheter aortic valve replacement: a systematic review and meta-analysis. Journal of the American Geriatrics Society, 2018. 66(12): p. 2417-2424.

10. Zaal, I.J., et al., A systematic review of risk factors for delirium in the ICU. Critical care medicine, 2015. 43(1): p. 40-47.

11. Koster, S., et al., Risk factors of delirium after cardiac surgery: a systematic review. European Journal of Cardiovascular Nursing, 2011. 10(4): p. 197-204.

12. Williams, S.T., Pathophysiology of encephalopathy and delirium. Journal of Clinical Neurophysiology, 2013. 30(5): p. 435-437.

13. Inouye, S.K., Delirium in hospitalized older patients. Clinics in geriatric medicine, 1998. 14(4): p. 745764.

14. Gao, W. and J. Jin, 1352: EARLY ONSET OF ICU DELIRIUM RELATED TO MORTALITY IN THE ICU AND HOSPITAL. Critical Care Medicine, 2020. 48(1): p. 653.

15. Sessler, C.N., et al., The Richmond Agitation-Sedation Scale: validity and reliability in adult intensive care unit patients. American journal of respiratory and critical care medicine, 2002. 166(10): p. 13381344.

16. Tadrisi, S., et al., RICHMOND AGITATION-SEDATION SCALE VALIDITY AND RELIABILITY IN INTENSIVE CARE UNIT ADULT PATIENTS; PERSIAN VERSION. 2009.

17. Siddiqi, N., A.O. House, and J.D. Holmes, Occurrence and outcome of delirium in medical in-patients: a systematic literature review. Age and ageing, 2006. 35(4): p. 350-364.

18. Inouye, S.K., et al., The short-term and long-term relationship between delirium and cognitive trajectory in older surgical patients. Alzheimer's \& Dementia, 2016. 12(7): p. 766-775.

19. Bellelli, G., et al., "Delirium Day": a nationwide point prevalence study of delirium in older hospitalized patients using an easy standardized diagnostic tool. BMC medicine, 2016. 14(1): p. 106. 
20. Arnulf, I., et al., REM sleep behavior disorder and REM sleep without atonia in patients with progressive supranuclear palsy. Sleep, 2005. 28(3): p. 349-354.

21. Fraigne, J.J., et al., REM sleep at its core-circuits, neurotransmitters, and pathophysiology. Frontiers in neurology, 2015. 6: p. 123.

22. Eikelenboom, P. and W.J. Hoogendijk, Do delirium and Alzheimer's dementia share specific pathogenetic mechanisms? Dementia and geriatric cognitive disorders, 1999. 10(5): p. 319-324.

23. Ljubisavljevic, V. and B. Kelly, Risk factors for development of delirium among oncology patients. General hospital psychiatry, 2003. 25(5): p. 345-352.

24. Bush, S.H., et al., Delirium in adult cancer patients: ESMO Clinical Practice Guidelines. Annals of Oncology, 2018. 29(Supplement_4): p. iv143-iv165.

25. Centeno, C., Á. Sanz, and E. Bruera, Delirium in advanced cancer patients. Palliative Medicine, 2004. 18(3): p. 184-194.

26. Aldemir, M., et al., Predisposing factors for delirium in the surgical intensive care unit. Critical care, 2001. 5(5): p. 265.

27. Sugimoto, M., et al., Pre-and intraoperative predictors of delirium after open abdominal aortic aneurysm repair. Annals of Vascular Diseases, 2015: p. oa. 15-00054.

28. Zhang, X.P., et al., High-fat treatment prevents postoperative cognitive dysfunction in a hyperlipidemia model by protecting the blood-brain barrier via Mfsd2a-related signaling. Molecular medicine reports, 2019. 20(5): p. 4226-4234.

29. Yıldızeli, B., et al., Factors associated with postoperative delirium after thoracic surgery. The Annals of thoracic surgery, 2005. 79(3): p. 1004-1009.

30. Siew, E.D., et al., Acute kidney injury as a risk factor for delirium and coma during critical illness. American journal of respiratory and critical care medicine, 2017. 195(12): p. 1597-1607.

31. Eriksson, I., et al., Urinary tract infection in very old women is associated with delirium. International psychogeriatrics, 2011. 23(3): p. 496-502.

32. Kassie, G.M., et al., Preoperative medication use and postoperative delirium: a systematic review. BMC geriatrics, 2017. 17(1): p. 298.

33. Neufeld, K.J., et al., Antipsychotic medication for prevention and treatment of delirium in hospitalized adults: a systematic review and meta-analysis. Journal of the American Geriatrics Society, 2016. 64(4): p. 705-714.

34. Lahariya, S., et al., Delirium in patients admitted to a cardiac intensive care unit with cardiac emergencies in a developing country: incidence, prevalence, risk factor and outcome. General hospital psychiatry, 2014. 36(2): p. 156-164.

35. Zhang, Z., et al., Prediction of delirium in critically ill patients with elevated C-reactive protein. Journal of critical care, 2014. 29(1): p. 88-92.

36. Alexander, S.A., et al., Interleukin 6 and apolipoprotein $E$ as predictors of acute brain dysfunction and survival in critical care patients. American Journal of Critical Care, 2014. 23(1): p. 49-57. 
37. McGrane, S., et al., Procalcitonin and C-reactive protein levels at admission as predictors of duration of acute brain dysfunction in critically ill patients. Critical care, 2011. 15(2): p. R78.

\section{Figures}
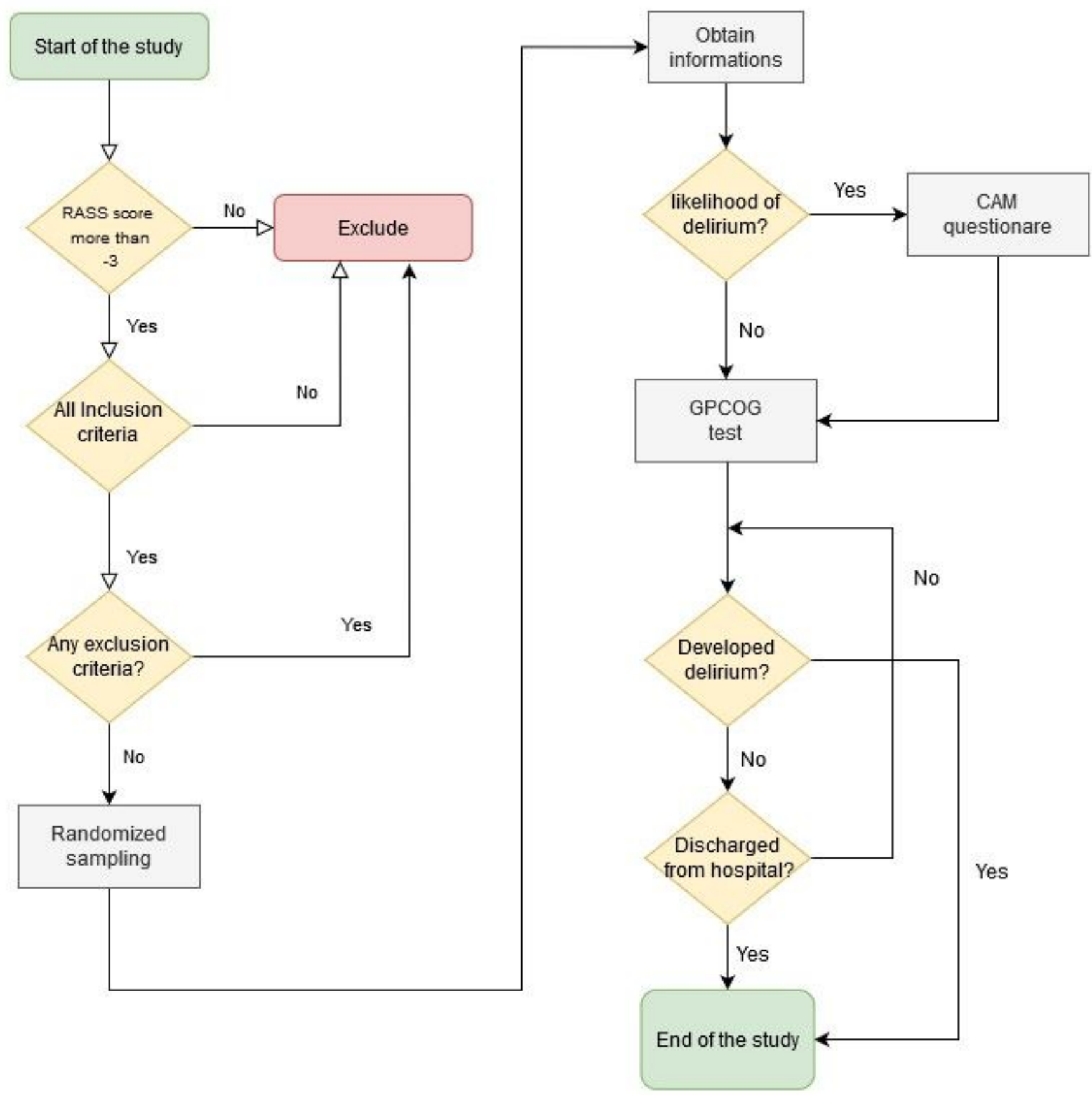

\section{Figure 1}

The flow chart of the study 